\title{
PRINCIPALES DILEMAS BIOÉTICOS EN LAS PERSONAS CON DISCAPACIDAD PROLONGADA
}

\author{
Laura Rueda Castro* \\ Orquídea Miranda Zúñiga**
}

\begin{abstract}
Resumen: Reconocida es a nivel mundial la dificultad para alcanzar la plena participación e integración social de las personas con discapacidad, situación que involucra valores éticos fundamentales. Un aporte importante será la aplicación de principios bioéticos orientadores del quehacer profesional bajo un nuevo paradigma.

Dilemas bioéticos como el consentimiento informado y la participación de la persona en la toma de decisiones que afectan su propia vida, implican para el profesional un desafío, la entrega de información adecuada y oportuna, el desarrollo de conductas profesionales que favorezcan la autonomía y el reconocimiento de estas personas como interlocutores válidos.

Del mismo modo ocurre cuando abordamos la sexualidad de las personas con discapacidad. Los profesionales deben permitir el desarrollo integral de las personas, reconociendo la sexualidad como un aspecto fundamental del ser humano, y frente al cual tienen derecho a ser orientados y educados tomando como base su historia biográfica y lo que cada uno desea de la vida (autodeterminación). Deben ser considerados por tanto, principios bioéticos fundamentales como la Justicia y la Autonomía.
\end{abstract}

Palabras clave: Principios bioéticos, discapacidad, consentimiento informado, sexualidad, integración

\section{MOST IMPORTANT BIOETHICAL DILEMMAS OF PEOPLE WITH LONG TERM DISABILITIES}

\begin{abstract}
The difficulty to achieve full participation and social integration of people with handicap is recognized at world level, situation that involves fundamental ethical values. The application of bioethic principles directed towards the professional chore under a new paradigm will signify an important contribution.

Bioethic dilemmas, such as informed consent and the person's participation in making decisions that affect his own life, imply a challenge, the delivery of appropiate and opportune information, the development of professional behaviors that will favor the autonomy and recognition of these people as valid speakers.

As to the subject of sexuality, professionals should allow everybody's integral development, recognizing sexuality as the human being's fundamental aspect, and faced to which you/they are entitled to be guided and educated taking into account their biographical history and what each one expects of his life (self determination). Therefore, they should be considered fundamental bioethic principles, such as Justice and Autonomy.
\end{abstract}

Keywords: Bioethical principles, disability, informed assessment, sexuality, integration

\section{PRINCIPAIS DILEMMAS BIOÉTICOS NOS POVOS COM DISCAPACIDADE PROLONGADA}

Resumo: É reconhecida em nível mundial as dificuldades que se impõem às pessoas portadoras de incapacidades para plena participação e integração social, o que envolve valores éticos fundamentais. Contribuição importante será obtida através de aplicação de princípios bioéticos que orientem a atividade profissional sob novo paradigma.

Dilemas como o consentimento informado e a participação de pessoas na tomada de decisões que afetam sua própria vida implicam em desafio para o profissional. De igual modo, o oferecimento da informação adequada e oportuna, o desenvolvimento de condutas profissionais que favoreçam a autonomia e o reconhecimento dessas pessoas como interlocutores válidos.

O mesmo ocorre quando tratamos da sexualidade de pessoas portadoras de incapacidades. Os profissionais devem permitir o desenvolvimento integral das pessoas, reconhecendo a sexualidade como expressão fundamental do ser humano, diante da qual têm direito à orientação e educação tendo como base sua história biográfica e o que cada um deseja para sua própria vida (autodeterminação). Assim sendo, devem ser levados em consideração princípios bioéticos fundamentais como a justiça e a autonomia.

Palavras chave: Princípios bioéticos, incapacidade, consentimento informado, sexualidade, integração

\footnotetext{
* Terapeuta Ocupacional.. Licenciada en Filosofía, Licenciada en Ciencia de la Ocupación, Magíster en Bioética. Correspondencia: ruedal@chi.ops-oms.org

** Terapeuta Ocupacional. Licenciada en Ciencia de la Ocupación. Master en Integración de Personas con Discapacidad. Correspondencia: omiranda@email.umayor.cl
} 


\section{Introducción}

Recibir la noticia de que nos encontramos (nosotros mismos o una persona cercana) frente a una situación de pérdida de habilidades o de alteración de las funciones psico - funcionales de nuestro organismo y de manera prolongada en el tiempo, constituye un momento difícil y doloroso. Las reacciones que acompañan a este vivenciar y el impacto de ellas dependerá de las características personales, culturales, religiosas y el estado económico social de las personas afectadas; pero existe en común, la misma resonancia afectiva conceptualizada como: pesar, pena, desmoralización.

De acuerdo con cifras de Naciones Unidas y Asociaciones Internacionales, existen entre 500 y 600 millones de personas en el mundo que presentan algún tipo de discapacidad física o mental, un $10 \%$ de los habitantes del mundo. Además, se estima que el $25 \%$ de la población total se ve afectada por las dificultades existentes para los discapacitados, por tratarse de familiares o responsables legales.

Asimismo, Naciones Unidas señala que un $98 \%$ de las personas discapacitadas que viven en países en desarrollo no tienen acceso a servicios de rehabilitación, que ningún país cuenta con sistemas de transporte totalmente accesibles, que sólo el $25 \%$ de los discapacitados cuenta con algún empleo o ingreso $o$, dicho de otra forma, que el $75 \%$ restante es considerado inactivo. Más aún, el $80 \%$ depende de sus familiares o amigos y cerca del $10 \%$ vive de la caridad o la limosna.(1)

En el marco de esta realidad mundial, se desarrollan diferentes programas de colaboración a la población portadora de discapacidades y la especialización de profesionales de la salud, cuyo principal foco de atención se dirige a la prevención (de otras discapacidades y de minusvalía) e integración social de estas personas. Esta tarea se involucra directamente con valores éticos como la defensa de la dignidad de las personas, el servicio, la solidaridad, entre otros. En este aspecto, el tema de la discapacidad se encuentra con el paradigma de la bioética, actual disciplina que reformula una ética de la vida para los tiempos de alta tecnología.

En este artículo, proponemos analizar los principales conflictos de tipo bioético que los profesionales de la salud enfrentamos en la tarea de la reinserción social de las personas discapacitadas. En primer lugar, abordaremos el tema de la discapacidad y, posteriormente, desde la visión de la bioética, expondremos los dilemas más habituales.

\section{La discapacidad permanente o prolon- gada}

Las reacciones vivenciales que experimentan las personas afectadas por la discapacidad son el pesar, la pena y la desmoralización. Con frecuencia los términos pesar y pena se usan indistintamente(2), cuando en verdad tienen significados diferentes. El pesar es el estado de haber sufrido una pérdida y experimentar muchas emociones y cambios significativos en la vida del individuo.

La pena es el proceso normal de reacción ante la pérdida. Se puede sentir pena ante la pérdida física de una persona o en respuesta a pérdidas de tipo social o simbólicas. Se puede sentir como una reacción mental, física, social o emocional. Las reacciones de tipo mental pueden incluir rabia, culpa, ansiedad, tristeza y desesperación. Las reacciones físicas pueden incluir trastornos en el dormir, cambios 
en el apetito, otras dolencias físicas. Las reacciones sociales pueden incluir los sentimientos que se experimenten en relación a tener que cuidar de otros en la familia, tener que abandonar responsabilidades con la familia, cambios en las condiciones del trabajo para el reintegro a él o, en ocasiones, tener que dejar el trabajo habitual.

La desmoralización es un fenómeno más complejo aún, la vive la persona afectada y también, por lo general, su familia; se trata de un cambio en la vivencia de existencia que cada persona percibe en su diario vivir. Se podría decir que es una especie de desmotivación hacia la participación en las actividades que hasta el momento de presentarse el problema eran placenteras o, por lo menos, cumplían con un objetivo claro y preciso que sustentaba la vida de la persona.

La desmoralización, desde su significado conceptual, nos indica falta o disminución de la moral. Ello conlleva la ausencia de actividad participativa en orden a lo social (incluyéndose lo social activo y pasivo) y la ausencia de interés en el otro ser humano que convive junto a él en la comunidad. La persona desmoralizada ( no es lo mismo que sin moral), al perder la iniciativa por satisfacer sus necesidades sociales y ejercer roles comunitarios, pierde al mismo tiempo las características que se le confieren como individuo de una especie que posee dignidad o, en otras palabras, que se encuentra capacitado para ejercer sus derechos y deberes que lo integran a un sistema organizado de usos sociales. La desmoralización tiene como producto más evidente el grave abandono de la autonomía de cada individuo, extremo que le lleva a adquirir las condiciones de un ente aislado, sin vinculaciones que lo ajusten a un todo con sentido.

El estado de desmoralización es diferente a sentirse aquejado de una depresión. En esta segunda diferenciación se distingue claramente una visión característica del mundo y de sí mismo:

- La consideración negativa de sí mismo: La persona muestra una marcada tendencia a considerarse como deficiente, inadecuada e inútil y atribuye sus experiencias desagradable a su defecto físico, moral o mental. Tiende a rechazarse a sí misma, ya que cree que los demás lo rechazarán.

- La consideración negativa del mundo: Se siente derrotada socialmente, considera al mundo como haciéndole enormes exigencias y presentándole obstáculos que se interfieren en el logro de los objetivos de su vida.

- La consideración negativa del futuro: Ve el futuro desde una perspectiva negativa y le da vueltas y más vueltas a una serie de expectativas negativas. El deprimido prevé que sus problemas y experiencias comunes continuarán indefinidamente y que se le amontonarán otros mucho peores en su vida.

La persona portadora de una discapacidad prolongada puede comprometerse afectivamente y presentar signos de depresión. La desmoralización, sin embargo, dependerá del talante emocional que cada persona posee como producto de su desarrollo biográfico (y axiográfico $\left.{ }^{1}\right)$.

Con respecto a la discapacidad congénita, podemos acotar que el desarrollo humano comienza desde el momento de la fecundación. De allí en adelante se desencadenan una serie de eventos que con el tiempo darán ori-

\footnotetext{
1 axio: valor, gráfico trayectoria temporal; correspondería a la historia personal donde se generan valores y se conforman escalas que fundamentan los patrones de comportamiento.
} 
gen a cada una de las partes que conforman nuestro ser. Sin embargo, esta serie de eventos es susceptible de errar, provocando anomalías en las estructuras que se están formando.

En la historia del hombre han existido muchos casos de malformación. En ocasiones, a estas personas se les ha considerado como monstruos, como el producto de un castigo divino o una maldición. Consecuentemente, como indignos de compartir con el resto de la comunidad siendo por ello maltratados, exiliados e incluso asesinados.

Hace 2400 años, en la antigua Grecia -casi al mismo tiempo que nacía la escuela hipocrática- los espartanos arrojaban desde el Monte Taigeto a todos los que presentaban algún trastorno físico, al parecer con fines más estéticos que eugenésicos. Hace sólo 60 años, los nazis -ahora sí con un objetivo totalmente eugenésico-eliminaron sistemáticamente a los discapacitados, especialmente mentales.

Entre ambos hitos, se ha tenido diferentes formas de enfrentar el tema de la discapacidad a lo largo de la historia de la "humanidad" (aunque esta palabra suene como irónica ante los hechos que estamos señalando), que han ido desde esas conductas criminales hasta actitudes totalmente opuestas, de protección e incluso adoración. No obstante, en la gran mayoría de los casos, no han sido justas ni favorables y la exclusión social ha sido la más habitual de encontrar en las diversas culturas y países.

Hoy se tiene conocimiento que estas anomalías son producto de la interacción de diversos factores a lo que, además, se ha agregado una visión de la discapacidad diferente: el reconocimiento de la importancia de la persona, de sus capacidades y de la trascendencia del entorno como factor condicionante de la integración. Esta visión, que plantea la importancia de la modificación del entorno (desde la barreras físicas y actitudinales) y la posibilidad de brindar apoyo personal e individualizado, ha constituido un paradigma que está siendo lentamente asumido por las propias personas con discapacidad, por sus familias y por los profesionales.

Ello se ve reflejado claramente en las Normas Uniformes promulgadas por Naciones Unidas y a las que ha adherido la mayoría de las naciones del globo.

Este cambio de concepción es fundamental. Sin embargo, aún debemos realizar esfuerzos importantes por trascender hacia la conducta profesional que marca de manera importante la conducta de la propia persona con discapacidad. En este sentido los valores y principios éticos adquieren relevancia y pueden ser una herramienta valiosa que ayude a los profesionales a revisar su hacer y propiciar el cambio de actitudes que ello requiere.

Si nos centramos en un ejemplo concreto, observamos que las amputaciones (congénitas o adquiridas durante el embarazo) son causadas por factores hereditarios, fármacos, enfermedades, exposición a radiaciones o traumatismos. Tradicionalmente, el concepto que se ha tenido, y que se mantiene hasta nuestros días, indica que las personas amputadas son discapacitadas y más que eso, minusválidas, en cuanto son incapaces de desarrollar una u otra actividad y de cumplir un rol social teniendo que depender de otros.

En este caso se debe tener presente la diferencia entre un amputado congénito y un amputado adquirido, tanto en la causa de su problema como en la forma de enfrentar su condición, lo que se refleja al profundizar su situación.

El discapacitado congénito, en sus primeros años, dado el desconocimiento de su si- 
tuación, no tiene problemas para desenvolverse con sus pares y satisface sus necesidades con las posibilidades que tiene. Hasta que se enfrenta a los prejuicios de la sociedad, en la que la familia y los profesionales están presentes, no comienza a cuestionar su condición, comparándose con el resto, sintiéndose diferente e incluso inferior; porque comienza a sobrevalorar actividades que no puede realizar o, simplemente, le cuesta llevarlas a cabo espontáneamente.

En el caso de los amputados adquiridos, su situación difiere de los anteriores en la forma cómo adquirieron tal condición ya que, siendo en un principio unas personas con autonomía y físicamente normales, experimentan la pérdida parcial o total de uno o más miembros, de manera traumática o como producto de una enfermedad. Para ellos la rehabilitación será diferente, ya que deben aceptar una condición discapacitante pasando a ser dependientes de otros para desenvolverse (por lo menos en un principio), mientras se amparen en un tratamiento, incluida la rehabilitación, que atenúe su alteración (entrenamiento protésico).

Se debe considerar que un amputado traumático y uno secundario a una enfermedad son también diferentes. Para el primero, el impacto o noticia de una amputación será muy mal acogida, pues, a veces, tiene en su memoria el accidente o parte de él, que relacionará y recordará con alguna situación, lugar, etc. Y mucho peor será si no recuerda nada. En el segundo caso, si bien no será bien acogido, puede consultarse previamente al paciente y, como también sabrá que esa determinación fue una alternativa a perder la vida, lo afrontará de mejor manera.

Ambos tipos de amputados, en un principio, "aceptan" su nueva condición como una "solución" ante la pérdida de la vida. Pero, de acuerdo con su desarrollo biográfico y axiográfico como ya se mencionó, tendrán diferentes formas de enfrentar su problemática transcurrido algún tiempo. Algunos de ellos pondrán todo de sí para asumir su nueva condición mientras que otros, estando en rehabilitación, se niegan a mejorar, cursando con depresión y deseando morir.

El problema mayor que afecta a estas personas, independiente de su actitud personal, es la gran cantidad de obstáculos que les presenta el mundo de hoy en los diferentes aspectos de la vida. Uno de estos problemas, y el que engloba a los otros, es el prejuicio que se tiene, como anteriormente se enunció, respecto de que ellos no son capaces de realizar una actividad concibiéndolos como "discapacitados". A la deficiencia que supone su problema anatómico, se le agrega la creencia de que son personas que no pueden hacer un trabajo de buena calidad. Incluso se llega a desconfiar, más que de su condición física, de su capacidad intelectual, privándoles de la posibilidad de utilizar sus talentos y potencialidades como cualquier persona.

Un ejemplo claro de marginación la constituye la arquitectura chilena, que hasta hoy ha sido diseñada para personas sin problemas de tipo físico, existiendo comodidades sólo para una parte de la población en la que se excluye, tanto a las personas con discapacidades como también a las personas adultos mayores, mujeres embarazadas o con niños, entre otras.

La arquitectura, así como la educación, la salud, y el mundo del trabajo, deben responder a cada uno de los habitantes con sus particulares condiciones físicas y mentales. Esto implica un conocimiento acabado de sus derechos, de sus posibilidades individuales y de sus capacidades, para lo que se requiere de un cambio de actitud y mentalidad de esta sociedad. Ello como solución primordial, aplicada a cada 
uno de los aspectos de la vida diaria de las personas.

Si bien es difícil, se debe formar en el seno de la familia actitudes positivas respecto de diversas situaciones como pueden ser: recibir el vuelto de unas manos sin sus dedos, enfrentar en la calle a personas que se comunican con sus manos o nos topan con sus bastones al desplazarse.

En este contexto, como equipo de salud, en primera instancia debemos generar un cambio de mentalidad en nosotros mismos y en la población, de tal forma que nos aboquemos, no sólo a prevenir un aumento de la discapacidad y/o minusvalía (prestando un servicio de rehabilitación parcial centrado en la persona), sino que también reconozcamos la importancia de generar cambios en el medio y brindemos atención personalizada, con apoyos individuales y personales que permitan lograr el gran objetivo de la rehabilitación integral: «la integración de estas personas en la sociedad.»

En Chile, la ley 19284 -que establece Normas para la Plena Integración Social de las Personas con Discapacidad-entrega un marco de referencia bastante completo respecto a lo que existe en otros países, especialmente en el área de Prevención, contando entre sus principios generales la igualdad de oportunidades y la no discriminación. Estos conceptos son claves como guía de los programas y prácticas, tanto en la promoción de la salud, como en los programas de salud y rehabilitación más tradicionales.

\section{Principales dilemas bioéticos}

Bioética es la ética de la vida, del ser humano como ser biológico que nace, vive y muere, del ser humano y su entorno social, lo que da origen a derechos y deberes en relación con ese ser viviente. La ética es una aplicación práctica de la filosofía. La bioética lleva la reflexión filosófica a un ámbito más preciso, más cerca de la aplicación en la vida cotidiana, en torno a valores fundamentales tales como: la búsqueda de la equidad, la autonomía y el respeto por las personas, la solidaridad, entre otros.

De allí que, para buscar una forma de acceso de las personas -desde una perspectiva ética- a los beneficios y riesgos de la "modernidad", surge hace cerca de cuarenta años, el movimiento de ética global o bioética, que incentiva el diálogo como forma de análisis y dispone de cuatro principios básicos para lograr ese propósito: la beneficencia, la nomaleficencia, la autonomía y la justicia.

En este punto, quisiéramos abordar los aspectos más íntimos de la relación entre el profesional de la salud y la persona afectada por una discapacidad que le produce desventajas en la toma racional de decisiones. El discapacitado pasa a constituirse en un ser humano más vulnerable, lo que hace necesario adoptar medidas especiales -desde un punto de vista ético- para proteger sus derechos como persona, ante decisiones que lo afectan en el ámbito de la salud. Específicamente en relación con tres temas de gran contenido ético: el consentimiento informado, aplicado en particular a quienes presentan discapacidades mentales o del sensorio, que les incapacitan parcial o totalmente para decidir por su propio juicio; la toma de decisiones, que podrían ser injustas, respecto del tratamiento de problemas de salud agregados que afecten a personas con discapacidades y el abordaje de la sexualidad de las personas con discapacidad, tema en el que se combinan los dilemas mencionados y los prejuicios sociales colectivos y personales de los profesionales. 


\section{Consentimiento informado del discapacitado}

En la relación clínica profesional (comprendiendo a profesionales de la salud que se rigen por códigos de conducta éticos) hay una confrontación de principios bioéticos que es preciso analizar, al abordar el tema del consentimiento informado. Fundamentalmente, el profesional se guía por el principio de la beneficencia y -primariamente- por el de la nomaleficencia, mientras que el paciente cuenta con el principio de autonomía, para decidir racionalmente lo que le es beneficioso.

La persona enferma, o que presenta algún grado de deficiencia, es el único decididor en las cuestiones relativas a su cuerpo. El médico u otro profesional del área no puede decidir sobre eso. "Lo único que puede hacer el médico es llevar la contraria al paciente cuando éste quiera hacer algo que atente contra los principios de no - maleficencia o de justicia, ya que esos dos principios son de rango superior y deben ser respetados aun en contra de la voluntad de los intervinientes. E incluso en esos casos no será el médico el que tome las decisiones, sino que el juez".(3)

En efecto, cuando se produce un conflicto ético que no logra ser resuelto por los dos interlocutores, ni por la mediación de un Comité de Ética, debe acudirse al juez para que decida, aun en contra de la voluntad del paciente o su familia (hace poco conocimos el caso de siamesas en Inglaterra en que se resolvió de esta forma).

Para que esta decisión por parte de la persona sea tomada, se requiere del conocimiento acabado de sus posibilidades y lo que cada una conlleva. Por tanto, tenemos que el procedimiento del consentimiento informado requiere dos elementos básicos: una información amplia y completa por parte del profesio- nal y una capacidad de decidir racionalmente ("competencia") por parte del paciente. Es indudable que ésta se ve afectada en muchos casos de discapacidad mental o del sensorio, que determinan la necesidad de crear una metodología de instrucción adaptada a la persona discapacitada o, en caso extremo, de un responsable legal que tome la decisión y de "un tercero" (normalmente el Estado, a través del poder judicial) que vele por los derechos de esa persona vulnerable.

\section{Participación en toma de decisiones en el tratamiento de la discapacidad}

Esta acción se encuentra directamente ligada al dilema de la aplicación del consentimiento informado y trata de incluir la consideración de la opinión de la persona afectada en la práctica de algún procedimiento terapéutico.

La necesidad de participación de individuo es la motivación para involucrarse activamente en los hechos que suceden alrededor del individuo. Se refleja, en este comportamiento, la necesidad de pertenencia al ambiente y tiene principal injerencia en la toma de decisiones, la necesidad de libertad se concretiza en la tendencia a decidir en forma autónoma a optar o no por alguna alternativa de acción. Esta facultad humana es la que debe protegerse en las personas vulneradas por la discapacidad y ésta es la misión ética de los profesionales que trabajan por atenuar la minusvalía.

La norma ética general para la aplicación o no de tratamiento ante un problema de salud establece que debe brindarse a todo aquél que lo necesite, en la medida de lo posible, intentando maximizar los beneficios y minimizar los riesgos. Por el principio de la autonomía el paciente podría rechazar un 
tratamiento, pero por el de la no-maleficencia, el profesional podría estar obligado a hacérselo. En esta confrontación de valores, a menudo actúan terceros (familiar responsable, por ejemplo) resolviendo el conflicto.

En los discapacitados por causa psíquica, los procesos psicopatológicos tienden a presentarse entre períodos de crisis y de estabilización. Muchos cuadros psiquiátricos se estabilizan, dejando a las personas afectadas en un estado defectual. La evolución de estos cuadros conlleva la recuperación de mayores grados de autonomía en la toma de decisiones. Los profesionales preocupados de brindar un servicio integral, deberán estar atentos a la recuperación de la autonomía y a su utilización concreta desde la apreciación de estas personas como interlocutores válidos frente a la toma de decisiones.

Del mismo modo esto debe aplicarse a discapacidades físicas, mentales o sensoriales y es el profesional el que debe fomentar la autonomía y la autodeterminación que le permita decidir respecto de temas fundamentales para su vida.

\section{Aspectos éticos de la sexualidad.}

La sexualidad es un tema relevante en todo ser humano y, como tal, también lo es en las personas con discapacidad. Sin embargo, éste es un aspecto que con frecuencia no forma parte de la intervención profesional y en el que se aplican muchas veces criterios y prejuicios personales.

La discapacidad en una persona, sea cual fuere su origen, requiere muchas veces de una adecuada orientación y educación en la esfera de la sexualidad ya que, de no suceder esto, las consecuencias podrían ser determinantes de mayor compromiso en las diferentes esferas de su vida.
Los personas con discapacidad manifiestan emociones e impulsos sexuales similares a los de los demás miembros de la sociedad, pero, como es lógico, con menos conocimientos. Esto, a su vez, hace que tengan menos posibilidades de resolver las diversas dificultades que en este sentido pueden surgir. Las familias y el personal especializado muchas veces dedican más tiempo a la educación y rehabilitación en sentido general, pero no tanto a la educación de la sexualidad.

"Cada individuo, no interesa cuál sea su discapacidad, tiene derecho al libre acceso a la información necesaria sobre la sexualidad $\mathrm{y}$, lo que es más importante, cada persona tiene derecho a la expresión de su sexualidad"(4)

El tema resulta muy discutido. Para muchos, las personas con discapacidad son seres que deben ser rechazados sexualmente. Lo cierto es que con independencia de su discapacidad son seres humanos y, por lo tanto, necesitan expresar sus sentimientos, sus impulsos. Ellos requieren, como es lógico, de un tratamiento y una educación especial, de acuerdo con el grado de afección que presenten.

El concepto de sexualidad, al igual que en todas las personas debe, en el caso de la persona con discapacidad, plantearse como parte integrante de la personalidad del individuo donde interactúan además factores de tipo social. Con independencia de una anomalía en lo biológico o de un impedimento físico, ese individuo crece y se desarrolla en sociedad, por eso hay que brindarle todo el apoyo que necesita para que su limitación no le impida realizarse en otras esferas de la vida y pueda expresar sus sentimientos y emociones.

En el caso de la discapacidad mental es importante destacar que las personas con déficit intelectual son muy sugestionables, lo 
que repercute en su actuación. Ellos no cuentan con suficiente capacidad para criticar las conductas $\mathrm{u}$ opiniones que les indican las personas que los rodean; ésta, entre otras, es una de las causas que pueden llevarlos a constituir un grupo vulnerable de abusos dentro de la sociedad.

El tema de la sexualidad en las personas con discapacidad posee sus propios prejuicios(5), los más habituales son los siguientes:

- Las personas con discapacidad son asexuadas.

- Las personas con discapacidad no se interesan en las relaciones sexuales.

- Los padres con discapacidad son una carga para sus hijos.

- Las personas discapacitadas no aprecian el valor de la vida.

- Todos las personas con discapacidad mental poseen exacerbados los impulsos sexuales

- Los personas con discapacidad dependen de los demás, son como niños, por lo que necesitan que se les proteja.
Los esfuerzos de los profesionales de la salud en el rol de orientadores y educadores de estos dilemas, deberán estar dirigidos a disminuir / abolir la potencia de estos prejuicios, centrándose en la mayor apreciación de los valores involucrados en el desarrollo de la intimidad (sexualidad) humana, aspecto frente al cual no existe discapacidad.

\section{Conclusión}

La consideración de valores y principios éticos fundamentales, constituye un punto de referencia que guiará el quehacer profesional, cuestión que se puede apreciar más claramente al enfrentar personas con grandes discapacidades. El valor de la persona humana, la consideración de principios bioéticos como la no maleficencia, beneficencia, justicia y autonomía, permitirán visualizar éstos y otros dilemas éticos que se presentan a diario en la práctica profesional. Ello contribuirá a fomentar la discusión de aspectos relevantes para las personas y sus familias y facilitará la equiparación entre el rol profesional y el de las personas a las que prestamos apoyo.

\section{Referencias}

1. Naciones Unidas. Dossier Informativo sobre las Normas Uniformes de las Naciones Unidas sobre la Igualdad de Oportunidades para las Personas con Discapacidad; 1998.

2. National Cancer Institute. Información en PDQ Sobre Cuidado Médico: Perdida, Pena, Pesar. [Sitio en Internet] Disponible en: http://www.cancer.gov/cancer_information/ Acceso el 18 de Julio 2002.

3. Gracia D. Bioética Clínica. Vol II Ética y Vida. Santa Fe de Bogotá: El Búho; 1998: 37.

4. Gordon S. Los Derechos sexuales de los discapacitados. La Habana: Taller de impresión ligera de la Federación de Mujeres Cubanas; 1981.

5. Coalition of Provincial Organizations of the Handicapped. Dispelling the Myths: Sexuality and Disabled Persons. Winnipeg; 1988. 